\title{
Lateral meningocele and defect of abdominal wall
}

\author{
Monica M. PlaczeK \\ M.B., Ch.B.
}

\author{
A. E. MacKinnON* \\ M.B. B.S., F.R.C.S.
}

The Neonatal Surgical Unit, Saint Mary's Hospital, Whitworth Park, Manchester M13 OJH

\section{Summary}

A case of lateral meningocele associated with deficiency of the thoraco-abdominal wall is reported. It is suggested that both of these defects are due to interference with the development of the paraxial mesoderm by a single injury.

\section{Introduction}

Lateral and anterior thoracic meningoceles are uncommon congenital malformations with the cyst

\section{Case history}

After an uneventful pregnancy a live male infanto was delivered at term by elective lower segment? Caesarean section, performed because of cephalo-iั pelvic disproportion. The mother was a primiparouso 23-year-old whose only medication throughout pregnancy had been iron. Both parents were healthy $\overrightarrow{\vec{t}}$ unrelated Muslims and there was no history of congenital malformation. The child required no음 resuscitation at birth, but multiple congenital ${ }^{-}$

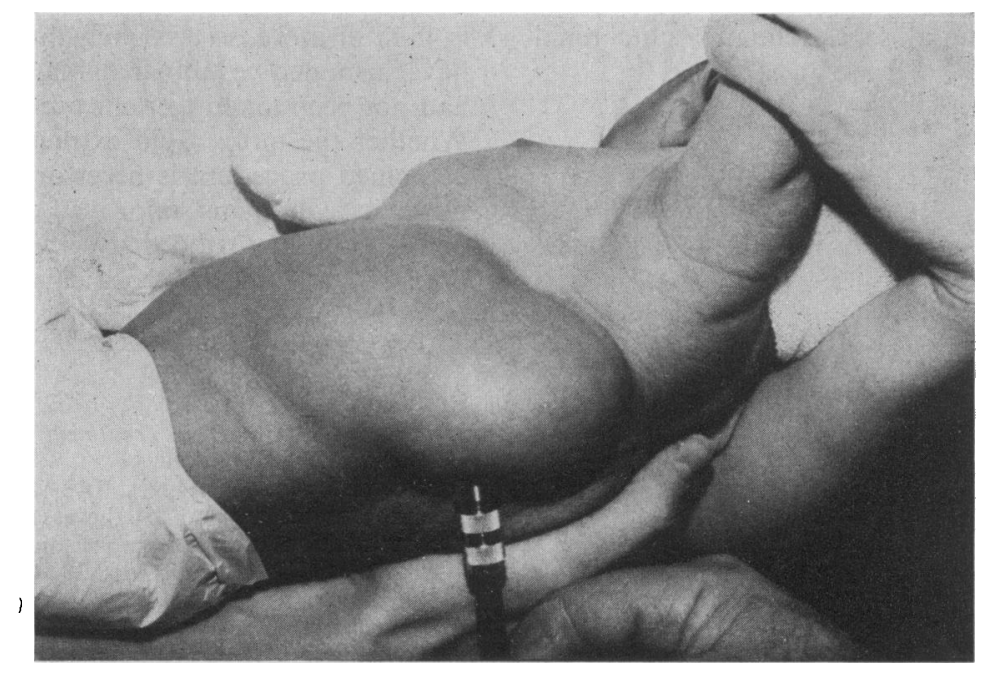

FIG. 1. Photograph of patient at birth.

usually lying within the thoracic cage. They are commonly associated with Von Recklinghausen's disease and skeletal malformation (Erkulvrawatr and Green, 1975).

Lateral meningoceles associated with defects of the abdominal wall are very rare (Goldman, 1949). One such case is the subject of this report.

*Correspondence: Mr A. E. MacKinnon, Sheffield Children's Hospital, Western Bank, Sheffield S10 2TH. malformations were immediately apparent. He was: therefore transferred to the Regional Neonatal Surgical Unit.

On examination the birth weight was $3260 \mathrm{~g}$ 尺 (30th centile) and the occipito-frontal circumferenceo $35 \mathrm{~cm}$ (50th centile). There were 2 swellings on the left side of the thoraco-lumbar region (Fig. 1). The? more posterior swelling was soft and cystic and transilluminated brightly. The anterior swelling, $\frac{}{\circ}$ 
which was completely reducible, extended from the lower costal margin to the iliac crest and medially to the midline, posteriorly it was related to the other cyst. There was a marked scoliosis in the lower thoracic region, concave to the left, and deformity of the thoracic cage with a reduction in the number of ribs. There was no evidence of neurological deficit, in particular in the lower limbs and anal sphincter. The external genitalia were normal. There were skin tags on the left ear and right side of the chest.

Routine laboratory investigations including haematology, biochemistry and bacteriology were normal. $\mathrm{X}$-ray of the trunk revealed a grossly disordered vertebral column with absence of the left half of each vertebra from the fifth thoracic to first lumbar bodies and a marked scoliosis (Fig. 2). The left sixth, seventh and eighth ribs were fused and the ninth to twelfth ribs on this side were absent. The lung fields appeared clear but the heart was displaced to the right. The intestine was seen to herniate through the left side of the abdominal wall. An intravenous pyelogram was normal.

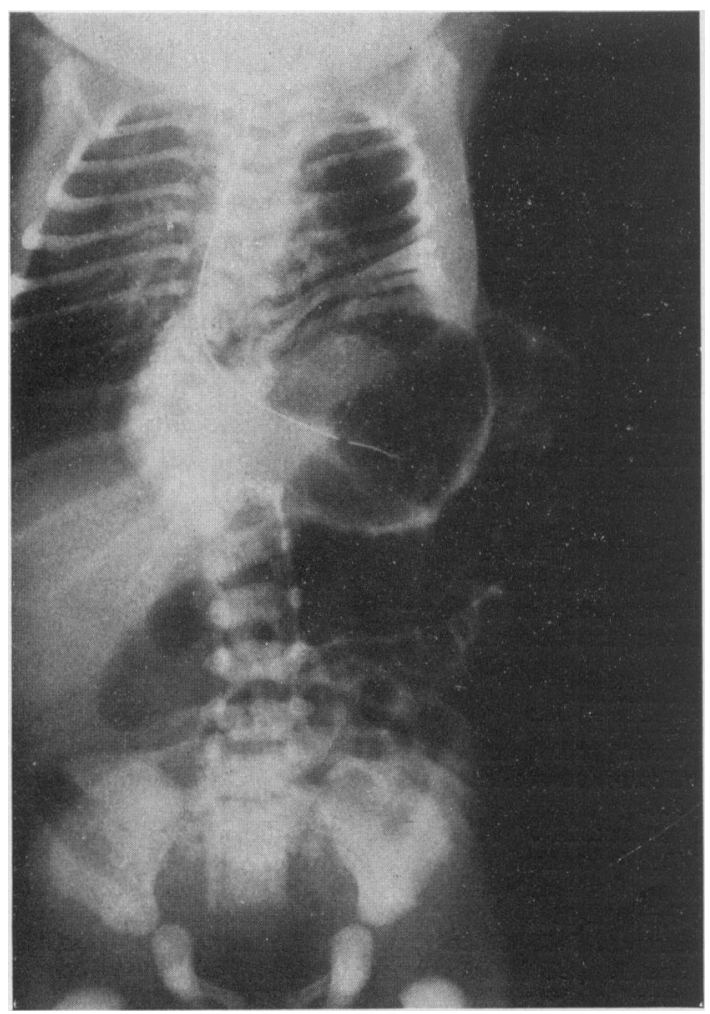

FIG. 2. X-ray of trunk at birth.
The infant progressed normally and on the fifteenth day of life the lateral thoracic swelling was explored under general anaesthesia. It was found to be a cyst measuring $4 \mathrm{~cm}$ in diameter and contained clear fluid. It arose from a narrow pedicle that was continuous with the dura and contained no visible neural elements. The cyst was excised and the dura sutured to obtain a watertight closure. Histological examination showed the wall of the cyst to be composed predominantly of fibrous tissue in which there were a few nerve fibrils and islands of rudimentary nervous tissue. On the inner lining of the sac there were remnants of ciliated columnar cells. There were no postoperative complications; in particular the occipito-frontal circumference remained on the 50 th centile. The child was discharged home at the age of 5 weeks.

At 7 months of age he was readmitted for surgery to the abdominal wall. His weight at that time was $6.5 \mathrm{~kg}$ (3rd centile) and the occipito-frontal circumference was $43 \mathrm{~cm}$ (30th centile). At operation it was confirmed that there was only a thin layer of fibrous tissue between the skin and peritoneum. This layer was plicated but in spite of this the hernia has since recurred.

At the age of 10 months he was able to stand holding on to support (Fig. 3) and all milestones appeared normal.

\section{Discussion}

During the second week of embryonic development the mesoderm is arranged in 3 longitudinal strips (Fig. 4). The most medial of these is called the paraxial mesoderm and becomes segmented. It gives rise to the sclerotome medially which in turn develops around the notochord and neural tube into the vertebrae and the dura mater. Laterally the paraxial mesoderm develops into the myotome or muscle plate which in turn form the muscles of the body wall. These muscles migrate into the unsegmented lateral mesodermal strip which in turn has split into the somatopleure and splanchnopleure. The third strip of mesoderm lies in an intermediate position and is known as the intermediate cell mass. This too becomes segmented and from it the urogenital system develops.

It is evident, therefore, that a single injury to the paraxial mesoderm may give rise to abnormalities of dura mater, vertebrae, ribs and body wall. If the damaging force extends to the closely related intermediate cell mass, then urogenital abnormalities are to be expected. Goldman (1949) reported a case of lateral thoracic meningocele associated with abnormalities of the thoracic cage on the right side and absence of the right renal tract.

Unilateral deficiency of the abdominal wall musculature is a rare abnormality caused by failure 


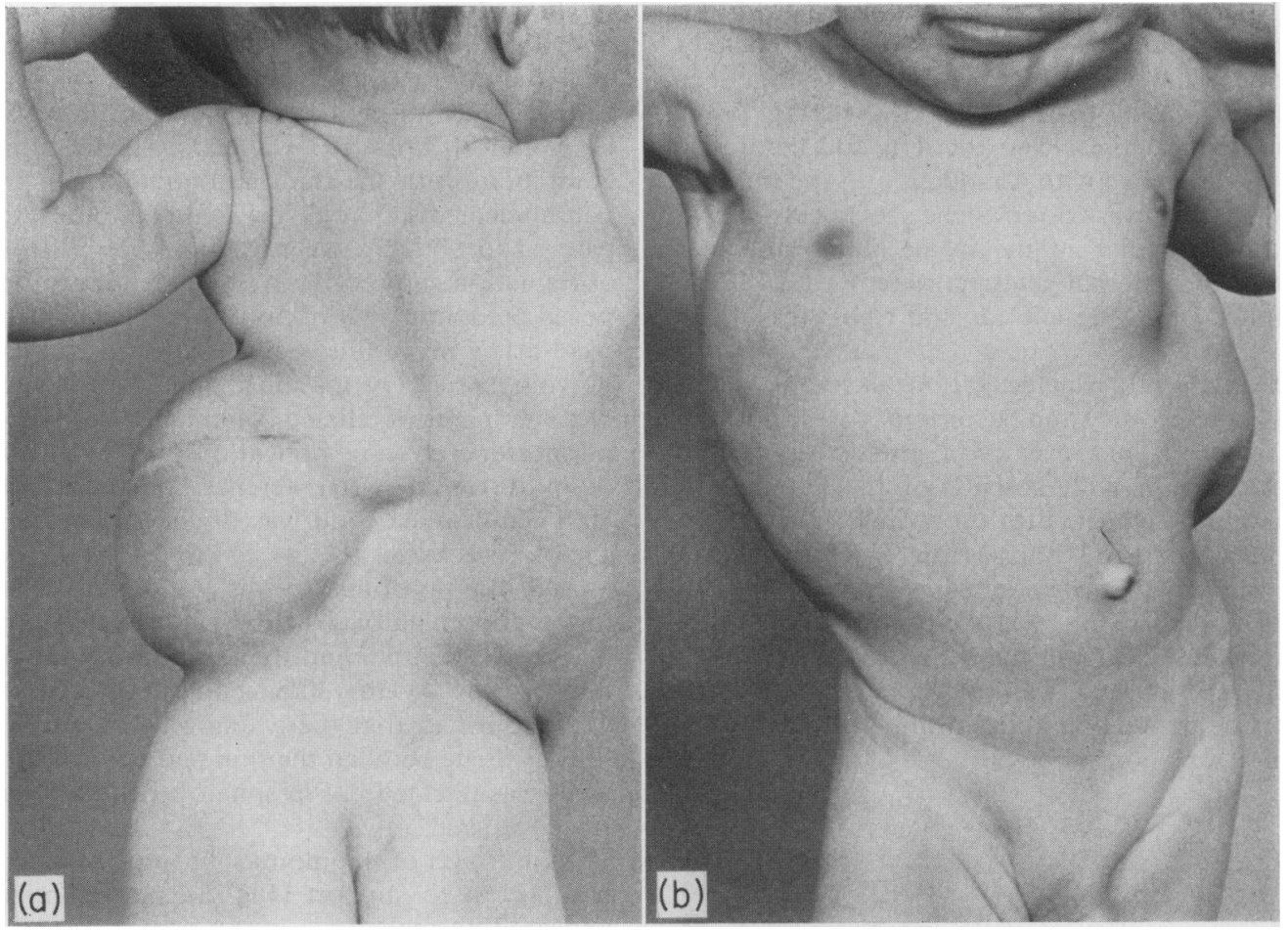

Fig. 3. (a) and (b) Photographs at 10 months of age.

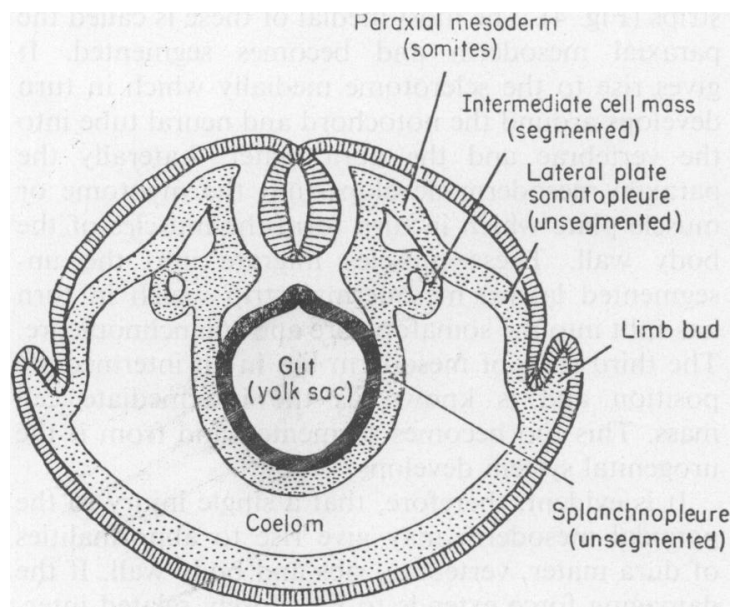

FIG. 4. Transverse section of embryo showing differentiation of embryonic mesoderm. Reproduced from Anatomy, Regional and Applied by R. J. Last (1977) 6th Edn p. 39 by courtesy of Churchill Livingstone, Edinburgh. of anterior growth of the muscle plate into theू somatopleure. More commonly it is bilateral ans part of the triad syndrome with dilatation of the renal tract and cryptorchidism. Alimentary ans skeletal abnormalities may also be present. This condition has been reported in association with के meningomyelocele of the lumbosacral area an $\dot{\delta}$ hydrocephalus (Mathieu et al., 1953).

\section{Acknowledgments}

The authors are grateful to Mr S. J. Cohen, consultanth surgeon, for his help in preparing this paper and allowing us to present his patient.

\section{References}

ERKulvRawatr, S. \& GREen, J.B. (1975) Intrathoracio meningocele associated with Von Recklinghausen's. disease (neurofibromatosis). Southern Medical Journal, 68 $\mathrm{G}$ 358.

GoLDMAN, I.R. (1949) Congenital malformation of vertebrae (hemivertebrae) with aplasia of corresponding ribsN associated with a lateral meningocele. Archives of Patho $\log y, 47,153$.

Mathieu, B.J., Goldowsky, S., Chaset, N. \& Mathiekb JR, P. (1953) Congenital deficiency of the abdomina muscles. Journal of Pediatrics, 42, 92. 